\title{
A POSSIBLE LAYER FOR THE TRANSMISSION OF FAST P WAVES FROM SURFACE DISTURBANCES*
}

\author{
By HARRY O. WOOD
}

UNLESS the principle of isostasy is rejected, in all its somewhat variant forms, there is some minimum depth beneath the surface of the land and sea, a relatively small depth, where the pressure due to the superincumbent rock, water, and atmosphere is the same everywhere.

The value of this least depth of equal pressure is not known exactly. Depending upon the data and methods employed (direct or indirect measurements of gravity or of deflections of the vertical) and of the hypotheses invoked (Pratt, or Airy, or combined mechanisms, and Hayford, Bowie, Heiskanen, or other modes of reduction), values have been obtained for the "depth of compensation" which range from something less than 60 kilometers to something more than twice this depth. And it is not known definitely whether these are outside limits.

However, the lesser of these depths is more than three times the best adjusted value for the usual depth of origin of local earthquakes in Southern California-where the rock surface ranges from below sea level (down to about 2,000 fathoms, say 3,700 meters) up to mountain heights greater than 10,000 feet (say, 3,000 meters) with a complicated pattern of topography and lithology -and it is more than twice the depth thus far found for those shocks in Southern California considered deeper than ordinary. Therefore, presumably, within the range of depth of the origins of these local earthquakes, and above, there may, and probably do, exist conditions of stress and consequent strain due to differences in load at such depths beneath different columns of equal area. At shallow depths such differences in pressure must be considerable. Over a small range of pressure at small depth the picture may be a very complicated one, over an area of regional extent. It should be simpler, and the pressure differences less, at greater depths. Thus, going downward we may pass through a depth-region of heterogeneity, both of rock material and of physical condition, so far as this is affected by pressure, at different places.

Also, passing downward from the earth surface, there is increase with depth not only of the pressure, but of the temperature as well. In detail, local conditions will cause variations in the rate and amount of these increases. In general, however, the increase in pressure will be less variable than the increase in temperature, especially at the lesser depths, as indicated by our relatively meager observations. Also, in most places not until a considerable depth has been reached will the temperature reach a significantly high value. But aside

* Manuscript received for publication, July 14, 1947. 
from strictly local conditions there is no reason to expect abrupt or rapidly changing increases in temperature-especially above a depth of, say, 20 to 30 kilometers.

Notwithstanding all this, except for unusual local peculiarities, it seems likely that at depths greater than a few kilometers average values of pressure and temperature may be used without introducing much error.

These increases in pressure and temperature produce changes in the density, $\rho$, ordinarily in opposite directions; also changes in the elastic constants, $\lambda$ and $\mu$, which are less simple and are, under the actual geological conditions (as distinguished from the controlled conditions of laboratory investigation), relatively little known. While our best guide toward their determination would seem to be the accurately controlled laboratory studies, it/must be emphasized that these neglect perforce the heterogeneity of the actual earth conditions, together with the gaseous and fluid components present in the rock material, either in solution or discretely, although the latter may have only slight effect on the over-all status.

These changes are such that, on the whole, over considerable ranges in depth, seismic wave speeds $-V_{\mathrm{P}}=\sqrt{\frac{\lambda+2 \mu}{\rho}}$ and $V_{\mathrm{S}}=\sqrt{\frac{\mu}{\rho}}$ - tend to increase with depth notwithstanding increase in the value of $\rho$. However, indication has been noted of a decrease in these speeds in a small range of depth in the neighborhood of 80 kilometers, attributed to a glassy state of rock material there as distinguished from a crystalline state at higher levels. If this is a fact, it seems possible that at a still higher level there may be similar variation in the rate of change of seismic wave speed due to other causes.

For natural earthquakes, especially ordinary local shocks, in the so-called "granitic" layer (that, neglecting sediments, above a depth of from 15 to 20 kilometers or less) values for $V_{\mathrm{P}}$ have been found repeatedly and from many widely separated localities in close agreement with the range 5.55 to 5.60 kilometers per second. For the Southern California region, evidence for this is very strong and abundant. Here, the first motion to arrive at stations out to more than 100 kilometers is considered to travel with such apparent velocities.

However, in all such determinations of wave speed both the location and depth of the origin and the origin time have to be "determined," that is, inferred, from the data of the seismometrically registered motion. We have no highly accurate independent determinations of these quantities, for we do not know when and where the shocks will occur and cannot plan to observe them critically. In such a connection, from studies of blasting where the location is known precisely, with the depth zero or at maximum a very shallow value, and the time of detonation known to a small fraction of a second, it has been found that "if the velocity is determined (in the present case) by the method unavoidably employed (in the former paper), namely, division of the difference 
in distance between the two stations by the time interval between the arrivals of the first motions, a value of 5.58 kilometers per second is obtained" (Wood and Richter, 1933, p. 102), whereas the wave speeds found directly from the definite, accurate data were $5.9+$ to $6.0-$ kilometers per second. Thus it remains possible that, with more abundant and more refined and accurate data and better and more refined methods, wave speeds in the "granitic" layer may in the future be determined which may differ slightly from those now considered to be best established. In the meantime, unless and until such changes are found, we must work with the values now considered best on the basis of a wealth of observations.

The whole range of values found is considerably greater than 5.55 to 5.60 kilometers per second; and it is known with certainty that the velocity of longitudinal waves in certain specific rock materials-anorthosite, for example-is higher, and in certain others lower, than the limits of the range given. Many detailed findings are listed by Macelwane (1939).

Studies of blasting elsewhere, as well as in Southern California, have disclosed waves which travel over distances of from, say, 25 to 300 kilometers with apparent velocities of about 6.0 kilometers per second. Other shallow disturbances of considerable energy, such as rock-bursts, also yield a similar velocity range. In the Victorville blast a value of $5.9+$ was determined very accurately at Pasadena at a distance from the source of 98.3 kilometers, and likewise a value of $6.0-$ at Riverside at a distance of 71.5 kilometers. The uncertainty in the value of the distances given is about $5 / 10$ kilometer in each instance (Wood and Richter, 1933). On several other occasions (data not yet published) apparent velocities of about this value have been found in Southern California with blasts at distances out to about 70 kilometers. Leet (1936) also has found values near 6.0 kilometers per second from blasts in Southern New England, and similar values have been found in such studies in Germany in the vicinity of Göttingen (Wiechert, 1929, and others, 1929 and 1931), as well as slightly higher values.

In the presentation of the study of the Victorville blast (Wood and Richter, 1933, p. 103 and pp. 108-109) a suggestion was made-but it was emphasized that it is geologically very improbable - that a body of rock buried not far below the surface, which has suitable areal distribution and thickness (perhaps thinness would be a better word) with suitable elastic properties and density, would serve to explain the abnormally high wave speed of the earliest registered motion, which was followed immediately by a phase of larger amplitude which had the usual apparent velocity of 5.5. In offering this suggestion, what was in mind was a body of rock of specific mineralogical composition-such, for example, as anorthosite-having the requisite velocity characteristics. Such rocks are known, but none of them are seen in the area involved and such information as is available indicates that they are absent from it, at least in 
bodies of any considerable size. The slightly higher wave speed, 6.0 - kilometers per second, is along the path from Victorville to Riverside, nearly at right angles to the structural trends and "grain" of the country; while the lesser, 5.9+ kilometers per second, is along a path from Victorville to Pasadena, which makes an angle of about $45^{\circ}$ with the regional trends and grain. Thus a body of rock of sufficient areal extent would be either large or oriented athwart the trend and grain at a large angle. While the presence of such a body of rock cannot be ruled out completely, it can be stated emphatically that no such body of rock is likely to be present. To assume that there is such a body definitely begs the question.

Since both the pressure and the temperature increase downward from the surface, beneath the increasing load and thickening blanket of the overlying rock, there is a possibility that the increasing pressure may bring about changes in the elastic constants and density sufficient to effect an increase in wave speed at a depth less than that where increased temperature will become high enough to offset this effectively. At still deeper levels, yet above the base of the "granitic" layer, although the pressure is still higher, the temperature may there have reached a value high enough to offset more effectively the action of the accompanying pressure and thus, possibly, cause a slight reduction in wave speed as compared with that immediately above-say to some value such that the average speed for the "granitic" layer as a whole becomes 5.55 to 5.6 kilometers per second. The thought suggested is that in passing downward from the surface, very near which the speed in "granite" is about 5.55 kilometers per second, often less, we may encounter and pass through a depthregion, or "layer," probably of subordinate thickness and possibly of vague upper and lower boundaries, in which a wave speed of 5.9 to 6.0 kilometers per second, or slightly more, is found -into a region where the speed, though higher than that near the surface, is lower than that in this immediately overlying layer. Thus this supposed variation in speed is considered due to general physical causes rather than specific changes in the mineralogical or lithological characteristics of the rock medium.

If there is such a higher-speed depth-region, or layer, at a relatively small to moderate distance below the surface, and well above the base of the "granitic" layer, it might well escape detection in studies of the instrumental records of local earthquakes, since unless it were of considerable thickness it would affect the wave paths and contribute to the wave speeds only slightly, since a major part of every ray emanating from the origin of a natural earthquake would be beneath the bottom of this supposed higher-speed layer, and another part above its top.

Until the general idea set forth above is considered and examined critically, there seems no point at all in making suppositions with respect to quantitiespressure, temperature, elastic properties, densities, depths, thicknesses-and 
then performing calculations to obtain wave speeds and ray paths. However, not until such numerical and geometrical relations can be determined on the basis of experiments and measurements will any such idea be either established on a satisfactory foundation or definitely dismissed from further consideration.

If the idea outlined has any merit, or if it should prove to be true in general, it nevertheless may be that such a higher-speed layer as is envisaged is not to be found beneath all parts of Southern California, or of any other regional area. For example, at some given depth beneath the mountainous areas with igneous crystalline rock at the surface, or other heavy rock, the pressure may be high enough to bring about the higher-speed condition, while at the same depth beneath some other lowlying areas underlain by thick unconsolidated sedimentary material, or deep water offshore, the pressure may not have become sufficient to bring about the changes in elasticity and density necessary to realize the higher speed.

Although beneath the surface the temperature gradient probably will not be the same under such diverse areas, the difference in the pressure effect will be a direct consequence of the unequal overlying loads, while any difference in temperature may be due to many complicating factors in addition to the blanketing effect. However, given time, such temperature variations will tend toward equalization because of heat conduction.

Thus it appears possible that any such shallow depth region of higher wave speed, if any exists, may be irregular both in the thickness of the layer and in its geographic disposition-thickening and thinning in an irregular way and perhaps absent altogether beneath some areas. Also-it may lie at different average depths in different places.

If such speculations have any actual basis in the upper part of the crust, the seismographic phases which should be expected in studies of major blasts and other large disturbances at or near the surface should exhibit differences and complexities as registered at different places, or when different disturbances, transmitted along different paths, are registered at the same place. But the effect of such a layer upon the seismograms of natural earthquakes registered in the same places should be comparatively slight for the reason given above.

If, now, we make the assumption that such a comparatively thin, irregular, and possibly patchy layer with higher wave-speed properties exists at a small mean depth, to establish this by field investigation would require the observation, with an adequate number of portable seismometers and the necessary auxiliary timing and other apparatus, suitably distributed and disposed, of a sufficiently large number of major blasts or other large surface disturbances set off at a considerable number of different points to furnish an adequately large number of ray paths in many different directions over different areas of the region studied. 
Such an investigation, therefore, would require a considerable assembly of specialized apparatus of research, the services of an adequate number of qualified persons during the work in the field, and either the coopperation of a large number of those who engage in major commercial blasting operations or of military experimentation on a large scale; or, far best, a large supply of explosives and permission to set off large amounts in desirable places would need to be available to the investigators. Because of all this, such field testing would be expensive at best, and unless the places and sizes of the explosions were controlled by the investigators themselves a very long time might, and probably would, elapse before sufficient data of a suitable kind could be collected. For it must be emphasized that data derived from the registration of natural earthquakes, originating at depths of $20 \pm 5$ kilometers, probably will not be of much aid in this, if, indeed, of any value at all-at least not until knowledge has been gained which will enable us to analyze and interpret the seismographic phases with much more certainty than now. And in any case it is an early arrival which must be established, earlier than expectable on the basis of present knowledge, or proof of a higher wave speed for $\overline{\mathrm{P}}$ than is now considered found.

Either the establishment of the reality of such a higher-speed layer at small depth, or the elimination of the possibility that this exists, would be of geophysical interest and, perhaps, importance. On the other hand, the effect upon local earthquake investigation would be of less importance. If established as real, it would necessitate only minor, perhaps negligible, corrections in the location of the epicenters and depths and a slight change in our views regarding wave paths and wave speeds in a small area surrounding the origin.

Further, for the sake of completeness in this statement, it must be pointed out that in the uppermost part of the crust there is much irregularity and heterogeneity in the rock material, mineralogically and lithologically, and in the size, shape, orientation, and distribution in place and depth of the bodies of rock of diverse kinds. Therefore, in highly detailed and refined studies by seismologic methods many small differences in wave speed and in the directions of wave paths must be expected, some of which might conflict confusingly with effects which might be looked for as due to pressure-temperature variation.

While the idea discussed here grew out of attempts to understand and explain an early wave phase- $\mathbf{P}_{6.0 \pm}$, - varying relations among pressure, temperature and diverse rock material might give rise to other depth-regions characterized by slightly different wave speeds.

The writer wishes to make acknowledgments to J. A. Anderson, L. H. Adams, B. Gutenberg, and C. F. Richter, who have read the manuscript and made critical suggestions, and to G. D. Louderback and P. Byerly also, but none of them should bear any responsibility for any shortcomings which remain. 


\section{REFERENCES}

WIECHERT, E.

1929. "Seismische Beobachtungen von Steinbruchsprengungen," Zeitschrift für Geophysik, 5: 159-162.

Brockamp, B., and $\mathrm{K}$. WOLCKen

1929. "Bemerkungen zu den Beobachtungen bei Steinbruchsprengungen," Zeitschrift für Geophysik, 5: 163-171.

Korte, WaLTER

1931. "Beiträge zur experimentalen Seismik," Zeitschrift für Geophysik, 7: 57-68.

Brockamp, Bernhard

1931. "Seismische Beobachtungen bei Steinbruchsprengungen," Zeitschrift für Geophysik, $7: 295-317$.

Wood, H. O., and C. F. Richter

1933. "A Second Study of Blasting in Southern California," Bull. Seism. Soc. Am., 23 (3): 95-110.

LEET, L. D.

1936. "Seismological Data on Surface Layers in New England," Bull. Seism. Soc. Am., $26(2): 129-145$.

Macelwane, James B., S.J.

1939. "Evidence on the Interior of the Earth Derived from Seismic Sources," chap. X (pp. 219-290) in Physies of the Earth, VII: Internal Constitution of the Earth (New York, McGraw-Hill Book Co., Ine.). 